\title{
Well-being in amyotrophic lateral sclerosis: a pilot experience sampling study
}

\author{
Ruben G. L. Real ${ }^{1 *}$, Thorsten Dickhaus ${ }^{2}$, Albert Ludolph ${ }^{3}$, Martin Hautzinger ${ }^{4}$ and Andrea Kübler ${ }^{1,5}$ \\ ${ }^{1}$ Department of Psychology I, Institute of Psychology, University of Würzburg, Würzburg, Germany \\ 2 Weierstrass Institute for Applied Analysis and Stochastics, Research Group "Stochastic Algorithms and Nonparametric Statistics," Berlin, Germany \\ ${ }^{3}$ Department of Neurology, University of Ulm, Ulm, Germany \\ ${ }^{4}$ Department of Clinical Psychology and Psychotherapy, University of Tübingen, Tübingen, Germany \\ ${ }^{5}$ Institute for Medical Psychology and Behavioural Neurobiology, University of Tübingen, Tübingen, Germany
}

\section{Edited by:}

Francesco Pagnini, Catholic

University of Milan, Italy

Reviewed by:

Michelle Dow Keawphalouk

Harvard/MIT, USA

Donatella Mattia, IRCCS Fondazione

Santa Lucia, Italy

Eleonora Volpato, Fondazione Don

Gnocchi, Milan, Italy

*Correspondence:

Ruben G. L. Real, Department of Psychology I, Insitute of Psychology, University of Würzburg, Marcusstr. 9-11, 97070 Würzburg, Germany e-mail: ruben.real@uni-wuerzburg.de
Objective: The aim of this longitudinal study was to identify predictors of instantaneous well-being in patients with amyotrophic lateral sclerosis (ALS). Based on flow theory well-being was expected to be highest when perceived demands and perceived control were in balance, and that thinking about the past would be a risk factor for rumination which would in turn reduce well-being.

Methods: Using the experience sampling method, data on current activities, associated aspects of perceived demands, control, and well-being were collected from 10 patients with ALS three times a day for two weeks.

Results: Results show that perceived control was uniformly and positively associated with well-being, but that demands were only positively associated with well-being when they were perceived as controllable. Mediation analysis confirmed thinking about the past, but not thinking about the future, to be a risk factor for rumination and reduced well-being.

Discussion: Findings extend our knowledge of factors contributing to well-being in ALS as not only perceived control but also perceived demands can contribute to well-being. They further show that a focus on present experiences might contribute to increased well-being.

Keywords: amyotrophic lateral sclerosis, ALS, coping, well-being, experience sampling, ESM, reminiscence, rumination

\section{INTRODUCTION}

Amyotrophic Lateral Sclerosis (ALS) is a rare neurodegenerative disease characterized by progressive paralysis (Logroscino et al., 2008). In the absence of a cure (Logroscino et al., 2008, 2010) the treatment of ALS focuses on the alleviation of symptoms and the maintenance of the best possible degree of functioning (Clarke et al., 2001). A rich literature exists on factors influencing quality of life and coping with the disease (c.f. Pagnini, 2013). For example, different styles of coping (Matuz et al., 2010; Montel et al., 2012a), spirituality (McLeod and Clarke, 2007), or care-giver relations (Chiò et al., 2004) have all been related to psychosocial well-being in patients with ALS. In line with psychological theories on subjective quality of life (e.g., Rapkin and Schwartz, 2004) the great physical burden of ALS shows no simple relationship to well-being, with many studies showing small and insignificant correlations (Robbins et al., 2001; Pagnini, 2013) or even positive associations (Lulé et al., 2009). However, many of the questionnaires used in research on well-being and quality of life in patients with ALS require participants to generate aggregate statements about their experiences, e.g., by asking them how they felt (on average) during the past days (Cohen et al., 1995; Robbins et al., 2001) or even weeks (Hammer et al., 2008). Thus, little is known about the factors which are associated with instantaneous well-being, i.e., well-being as it fluctuates from moment to moment throughout the day.

In this study, therefore, the experience sampling method (ESM) was used (Larson and Csikszentmihalyi, 1983; Stone et al., 2003) to analyze possible predictors of well-being. Experience sampling, originally developed because of concerns of how accurate people are in "reconstructing their experience[s] after the fact" (Larson and Csikszentmihalyi, 1983, p. 42), asks participants to record their experiences directly at the moment of sampling. By randomizing sampling times throughout the study period, it is possible to collect representative data on the participants' experiences, such as well-being, and possible influencing factors (Shiffman et al., 2008; Scollon et al., 2009).

At its most basic level, flow theory assumes that the physical and mental activities people engage in, influence their well-being. More specifically, it predicts that well-being is highest if perceived control and perceived demands match in a given situation (Csikszentmihalyi, 1990). Any imbalance between demands and abilities results in reduced well-being, e.g., feelings of frustration, when demands exceed abilities, or of boredom, when abilities exceed situational demands (Csikszentmihalyi, 1990). Given that the progressive loss of motor functions requires constant adaptation and change (King et al., 2009), we hypothesized that 
finding this balance would be of crucial importance in patients with ALS. A second prediction of flow theory is that well-being depends on the extent to which thoughts are focused on present experiences (Schmidt et al., 2007). However, the little data available from patients with ALS is heterogeneous. Support comes from a study by Plahuta et al. (2002), which found that hopelessness, a future oriented direction of thought, is related to increased suffering and higher intentions of suicide in patients with ALS. However, the use of the coping strategy "planning" (Carver, 1997), an arguably more direct and less negatively valenced indicator of "thoughts directed toward the future" does not seem to be strongly related to well-being (Montel et al., 2012b). Qualitative findings from interviews with patients are also mixed (Fanos et al., 2008). On the one hand, several subjects indicated the use of "living in the moment" as a successful coping strategy while others sought consolation in remembering positive past experiences. The constant adaptation and change required for coping with ALS (King et al., 2009), necessitates disengaging from goals no longer attainable, or anticipating disengagement from goals which may become unattainable in the future. Generally, confrontation with unattainable goalseven more so when the factors which make a goal unattainable are essentially uncontrollable as is the case in ALS (NolenHoeksema et al., 2008) - has been shown to be a risk factor for rumination, and, thus, decreased well-being (Nolen-Hoeksema, 1991; Martin and Tesser, 1996). Therefore, we hypothesized that thinking about the past and future would be associated with reduced well-being, and that rumination would mediate this effect.

\section{METHODS}

\subsection{PATIENTS}

A convenience sample of ten patients (seven male; see Table 1) fulfilling the revised El Escorial criteria for clinically definite ALS (Brooks et al., 2000) was recruited via the Institute for Medical Psychology and Behavioral Neurobiology of the University of Tübingen and the Department of Neurology of the University of Ulm. All participants contacted for inclusion agreed to participate in this study. Mean time since diagnosis was 38.10 months $(S D=35.96$, range $=12-129)$. One patient had a percutaneous endoscopic gastrostomy (PEG), and three were non-invasively ventilated. The study was approved by the Ethical Review Board of the Medical Faculty, University of Tübingen. Written informed consent was obtained from all participants.

Table 1 | Patients' Characteristics.

\begin{tabular}{lcccccc}
\hline & ALSFRS-R & ADI-12 & well-being & $\boldsymbol{M}$ & SD & Range \\
\hline Age (years) & \multirow{2}{*}{0.52} & 0.32 & -0.09 & 52.30 & 11.56 & $35-71$ \\
ALSFRS-R & & $0.59^{\#}$ & $-0.75^{*}$ & 19.20 & 10.57 & $1-35$ \\
ADI-12 & & & $-0.63^{\#}$ & 20.70 & 7.41 & $12-34$ \\
well-being & & & & 4.79 & 0.69 & $3.93-5.86$ \\
\hline
\end{tabular}

Intercorrelations, means, and standard correlations.

Note: $N=10 .{ }^{*} p<0.10,{ }^{*} p<0.05$.

\subsection{PROCEDURE}

We collected data on well-being, perceived demands, perceived control, temporal direction of thoughts and rumination over the course of two weeks at three randomly chosen times per day using the ESM. Patients were provided with a pager that beeped when they should record their experiences using a standardized questionnaire. To avoid interference with morning and evening caring routines, sampling was restricted to between 10 a.m. and 6 p.m. Patients were instructed to answer the ESM-questionnaires immediately after the beep and while answering the questions to refer only to the situation in which the beep occurred. At the beginning of the two weeks study period, patients were visited at their homes and the procedure was explained in full detail and questions concerning the study protocol were answered.

\subsection{MEASURES}

Functional status was assessed with the revised ALS Functional Rating Scale (ALSFRS-R) (Cedarbaum et al., 1999), and the extent of depressive symptoms with the ALS specific ALS Depression Inventory (ADI-12) (Hammer et al., 2008). These standardized measures were collected once at the beginning of the two week study period.

The ESM-questionnaire used in this study was previously used by Csikszentmihalyi and Larson (1987) and assessed the extent of perceived demands and control with two items, "How challenging was your activity?" and "Was the situation under your control?" To minimize participants' burden in terms of time and effort, rumination was measured with the single item "Did your thoughts turn round in circles?” (Jong-Meyer et al., 2009) and temporal direction of thoughts with two face-valid items ("Did you look ahead/to the past?"). Each item was scored on a 10point Likert-type scale ranging from 0 (low) to 9 (high). Patients' well-being was measured with 13 well-being-related items developed by Larson and Csikszentmihalyi (1983). Each item was scored on a 7-point Likert-type scale, and a composite score reflecting well-being was derived by averaging ESM-items of the well-being category. To determine the reliability of this scale while taking the inter-correlation of repeated measurements into account Cronbach's $\alpha$ was calculated across subjects for every time point during the 2 -week study period, yielding 42 coefficients. Reliability of the well-being scale was satisfactory (median $\alpha=0.86$ ).

Patients completed an average of 33.30 assessments (range 13-43). Fifty percent of all questionnaires were answered within $10 \mathrm{~min}$ and $75 \%$ within $45 \mathrm{~min}$ after the beep. Further analysis revealed that without the most disabled participant (one male who used a personal computer to answer the questionnaires) $75 \%$ of all questionnaires were answered within 30 min after the beep. One patient was excluded from the following analyses because of excessive numbers of missing items, leading to a final sample size of $N=9$.

\subsection{STATISTICAL ANALYSES}

Random intercept multiple regression analysis was used to test the hypothesis that well-being depended on the balance of demands and control (Edwards and Cooper, 1990; Edwards, 1994, also see Supplementary Material). The analysis was also performed with 
functional status included as a covariate, which indicated that the results we report here were independent of functional status. Mediation analysis (MacKinnon et al., 2002; Kenny et al., 2003) was used to test the hypothesis that thinking about the past would have a negative effect on well-being and that this effect would be mediated by rumination. We report $95 \%$ confidence intervals for the mediated effect (Tofighi and MacKinnon, 2011). The same analysis was repeated for thinking about the future. Analyses were performed in R (R Development Core Team, 2011). Cohen's $f^{2}$ was calculated as a measure of the effect size (Selya et al., 2012).

\section{RESULTS}

Average well-being (see Table 1) was marginally negatively associated with symptoms of depression, but significantly positively associated with functional status. Functional status was marginally positively associated with symptoms of depression. Patient's age was not associated with functional status, symptoms of depression, or well-being.

Our first hypothesis suggested that well-being would depend on the balance of perceived control and demands. However, no support was found for this prediction (see Supplementary Material). Instead, results indicated that perceived control was associated with increased well-being $(b=0.18, S E=0.03, p<$ $0.001, f^{2}=0.22$ ), regardless of the level of perceived demands. In contrast, the effect of perceived demands depended on whether these demands were perceived as controllable: Only, if demands were perceived as controllable, they were associated with increased well-being $\left(b=0.09, S E=0.02, p<0.001, f^{2}=\right.$ $0.17)$. If demands were perceived as uncontrollable, the positive effect of demands was significantly reduced $(b=-0.14$, $\left.S E=0.05, p<0.01, f^{2}=0.03\right)$, rendering the effect of demands on well-being non-significant $(b=0.02, S E=0.05, p>0.70$, $\left.f^{2}=0.01\right)$.

Our second hypothesis proposed that thinking about the past would lead to increased rumination which in turn would reduce well-being. Table 2 shows that thinking about the past (Row 1), thinking about the future (Row 2), and rumination (Row 3 ) were associated with lower well-being, and that thinking about the past (Row 4) was significantly and thinking about the future (Row 5) was marginally associated with higher rumination. Mediation analysis confirmed a negative effect of thinking about the past on well-being via rumination ( $b=-0.03,95 \%$ CI $[-0.05-0.02])$. No evidence was found that the negative effect of thinking about the future on well-being was mediated via increased rumination $(b=-0.01,95 \%$ CI $[-0.020 .001])$.

\section{DISCUSSION}

Little is known about how patients with ALS cope with their disease from moment to moment in daily life. Thus, in this study the ESM was used to record patients' experiences throughout the day. Despite their severe physical disability, patients were able to complete the ESM-questionnaires within a short time after the scheduled signal. These results provide evidence that even a potentially demanding procedure such as the ESM may be a valuable tool in assessing patients' thoughts, well-being and cognitive state. The relationships between functional status, presence of depressive symptoms and well-being indicated that depressive symptoms were more present and well-being was lower in early stages of the disease. This result mirrors findings of better quality of life in patients who are longer affected by the disease (Lulé et al., 2008, 2009).

Flow theory predicts that well-being depends on finding the optimal balance between perceived demands and control (Csikszentmihalyi, 1990), and we hypothesized that finding this balance would be of crucial importance in patients with ALS. However, our data did not support this hypothesis. Instead, but in line with previous findings (Plahuta et al., 2002), perceived control was associated with increased well-being, regardless of the level of perceived demands. However, our results extend this idea, by showing that increased demands can also contribute to increased well-being, as long as they are perceived as controllable. While flow theory is primarily concerned with experiences related to activities in the present, experiences may also differ in their temporal orientation. We hypothesized that if attention is not focused on the here and now, but directed toward the past or the future, this would increase the risk for rumination, which in turn would reduce well-being. Our results indicate that thinking about the past was indeed associated with decreased well-being and that this effect was mediated by rumination. While some patients may find solace in remembering the good times they have had (Fanos

Table 2 | Results of mediation analysis.

\begin{tabular}{|c|c|c|c|c|c|c|c|c|}
\hline & Predicted & Predictor & $\boldsymbol{b}$ & $S E$ & $D F$ & $t$ & $\boldsymbol{p}$ & $f^{2}$ \\
\hline \multicolumn{9}{|c|}{ PREDICTORS OF WELL-BEING } \\
\hline 1 & Well-being & Thinking about the past & -0.03 & 0.01 & 250.83 & -2.73 & 0.01 & 0.02 \\
\hline 3 & Well-being & Rumination & -0.08 & 0.01 & 255.84 & -5.60 & $<0.001$ & 0.10 \\
\hline \multicolumn{9}{|c|}{ PREDICTORS OF RUMINATION } \\
\hline \multicolumn{9}{|c|}{ MEDIATION MODELS } \\
\hline 6 & Well-being & Thinking about the past & 0.00 & 0.01 & 247.77 & 0.17 & 0.87 & \\
\hline 7 & & Rumination & -0.08 & 0.02 & 254.12 & -4.79 & $<0.001$ & \\
\hline 8 & Well-being & Thinking about the future & -0.02 & 0.01 & 250.30 & -1.66 & 0.10 & \\
\hline 9 & & Rumination & -0.08 & 0.01 & 254.83 & -5.43 & $<0.001$ & \\
\hline
\end{tabular}


et al., 2008), reminiscing may also introduce the risk of being reminded of unattainable goals, thus, in turn, increasing the risk of reduced well-being. Likewise, thinking about the future was negatively associated with well-being; however, it was only loosely related to rumination, and no support was found for rumination mediating a negative effect of thinking about the future on wellbeing. We may thus speculate that thoughts about the future are not necessarily ruminative, possibly only in patients experiencing hopelessness (Plahuta et al., 2002).

\subsection{LIMITATIONS AND CONSIDERATIONS FOR FUTURE STUDIES 4.1.1. Sampling}

Several limiting factors of the current study deserve attention. To minimize interruption of daily routine, time slots in which patients had pre-set appointments (e.g., occupational therapy) were excluded from the experience sampling and sampling was restricted to the time between 10 a.m. and 6 p.m. This restriction may have prevented us from sampling several activities of daily living, e.g., personal hygiene, which may be very demanding and frustrating for severely ill patients (Foley et al., 2014).

\subsubsection{Economic considerations}

From a research perspective it is often desirable to use longer rather than shorter instruments, if only to increase reliability (Spearman, 1910). However, in a repeated measures context, this desire needs to be balanced with economic considerations, especially in a sample in which motor difficulties, e.g., when holding a pen, are common. Thus, to avoid spurious results, e.g., low well-being due to the frustrating experience of having to repeatedly answer a long questionnaire, and to reduce the risk of high levels of non-reponse (Iglesias and Torgerson, 2000) the questionnaires we used were kept as short as possible. Yet it is clear, that assessing rumination with only one item is a simplification of a complex construct. However, both empirical (Jong-Meyer et al., 2009) as well as theoretical accounts (Carver, 1996) suggest, that the item we used possesses high face validity for assessing rumination.

\subsubsection{Sample size and generalizability}

Finally, although not uncommon in ESM studies (e.g., Teuchmann et al., 1999), the small sample size limits generalization of results, until future studies can replicate our findings. Further, our analyses focused on within-subject variables, but between-subject factors and their interactions could also play an important role. For example, care-givers tend to underestimate the patient's quality of life (Trail et al., 2003), and it might be interesting to know, whether this is accompanied by a tendency to shield the patient from demanding activities, which according to our findings, might actually improve the patient's well-being. To the best of our knowledge, our study is the first to employ the ESM in patients with ALS. Thus, little information on appropriate sample sizes was available beforehand, demonstrating the pilot character of this study. Judging from the obtained effect sizes, mainly small to medium according to Cohen (1992), we suggest increasing sample sizes in future studies. Results from simulation studies (Snijders and Bosker, 1993) suggest, that an increase in the number of participants might be of greater interest than increasing the number of observations per participant. Further, if a research question focused primarily on between-subject factors (see above) a larger sample size would also be recommended. Thus, it should be kept in mind, that the optimal balance between number of participants and the number of observations per participant is dependent on the particular research question.

The ESM methodology has been applied in a variety of chronic diseases, e.g., in patients with cancer, chronic pain, cardiovascular diseases, or patients requiring hemodialysis (c.f. Smyth and Stone, 2003), testifying to the wide applicability of the method (c.f. Christensen et al., 2003). However, depending on the research questions, sophisticated data analysis methods, and technical equipment, e.g., electronic paging and recording devices with a user-friendly and unobstrusive interface, may be needed. Given the large challenge of living with ever progressing motor impairments, we suggest that future studies may take full advantage of the widespread availability of smartphones which lend themselves to ESM-studies (e.g., Runyan et al., 2013). Using such a system it might even become possible to densely track the evolution of coping with ALS over prolonged periods of time. However, apart from generating valuable data for research purposes, such a procedure might also affect patients' well-being, e.g., by making relations between certain situations and well-being transparent to the patient.

\subsection{SUMMARY}

Our results suggest that the ESM may be a valuable tool to elucidate components of coping and well-being in daily life in patients with ALS. Perceived control over challenging situations seems to be of major importance for maintaining high wellbeing. We further show that well-being may not only depend on perceived control, but that it also depends on the level of perceived demands. Encouraging patients to pursue, within limits, potentially demanding activities might help to improve their well-being.

Both thoughts directed toward the past and the future may negatively affect well-being, whereas rumination mediates this effect when thoughts are directed toward the past. We speculate that patients who experience poor well-being and may even be depressed might benefit from interventions which not only strengthen an internal locus of control (Nonnenmacher et al., 2013; Foley et al., 2014) but also encourage living in the present.

On a more global perspective the flow-theoretical approach and our results correspond well with recent work aiming at evaluating mindfulness-based (Kocovski et al., 2009) interventions in patients with ALS (Pagnini et al., 2014a,b). These interventions aim to help focusing on the experiences in the here and now, and thus to reduce the risk of reactive, e.g., ruminative, thoughts, which may otherwise reduce well-being or even lead to depression (Bishop et al., 2004). Our results provide further evidence for this promising approach, by highlighting the importance of the temporal direction of thoughts and the importance of perceived demands and control for the experience of well-being in patients with ALS. 


\section{FUNDING}

Funding for this study was provided by a grant to Ruben G. L. Real of the research training group 1253/1 which is supported by the German Research Foundation (Deutsche Forschungsgemeinschaft, DFG) by Federal and Länder funds. The study sponsor had no role in the study design, collection, analysis or interpretation of the data, writing the manuscript, or the decision to submit the paper for publication.

\section{ACKNOWLEDGMENTS}

We thank Petra Nedele who assisted in data collection. This publication was funded by the German Research Foundation (DFG) and the University of Wuerzburg in the funding programme Open Access Publishing.

\section{SUPPLEMENTARY MATERIAL}

The Supplementary Material for this article can be found online at: http://www.frontiersin.org/journal/10.3389/fpsyg.2014. 00704/abstract

\section{REFERENCES}

Bishop, S. R., Lau, M., Shapiro, S., Carlson, L., Anderson, N. D., Carmody, J., et al. (2004). Mindfulness: a proposed operational definition. Clin. Psychol. Sci. Pract. 11, 230-241. doi: 10.1093/clipsy.bph077

Brooks, B. R., Miller, R. G., Swash, M., Munsat, T. L., and for the World Federation of Neurology Research Group on Motor Neuron Diseases. (2000). El escorial revisited: revised criteria for the diagnosis of amyotrophic lateral sclerosis. Amyotroph. Lateral Scler. Other Motor Neuron Disord. 1, 293-299. doi: 10.1080/146608200300079536

Carver, C. S. (1996). "Goal engagement and the human experience," in Ruminative Thoughts, Volume IX of Advances in Social Cognition, ed R. S. Wyer (Mahwah, NJ: Lawrence Erlbaum), 49-59.

Carver, C. S. (1997). You want to measure coping but your protocol's too long: consider the brief cope. Int. J. Behav. Med. 4, 92-100. doi: 10.1207/s15327558ijbm0401_6

Cedarbaum, J. M., Stambler, N., Malta, E., Fuller, C., Hilt, D., Thurmond, B., et al. (1999). The alsfrs-r: a revised als functional rating scale that incorporates assessments of respiratory function. J. Neurol. Sci. 169, 13-21. doi: 10.1016/S0022-510X(99)00210-5

Chiò, A., Gauthier, A., Montuschi, A., Calvo, A., Di Vito, N., and Mutani, R. (2004). A cross sectional study on determinants of quality of life in als. J. Neurol. Neurosurg. Psychiatry 75, 1597-1601. doi: 10.1136/jnnp.2003.033100

Christensen, T., Barrett, L., Bliss-Moreau, E., Lebo, K., and Kaschub, C. (2003). A practical guide to experience-sampling procedures. J. Happiness Stud. 4, 53-78. doi: 10.1023/A:1023609306024

Clarke, S., Hickey, A., O’Boyle, C., and Hardiman, O. (2001). Assessing individual quality of life in amyotrophic lateral sclerosis. Qual. Life Res. 10, 149-158. doi: 10.1023/A:1016704906100

Cohen, J. (1992). A power primer. Psychol. Bull. 112, 155-159. doi: 10.1037/00332909.112.1.155

Cohen, S. R., Mount, B. M., Strobel, M. G., and Bul, F. (1995). The mcgill quality of life questionnaire: a measure of quality of life appropriate for people with advanced disease. a preliminary study of validity and acceptability. Palliative Med. 9, 207-219. doi: 10.1177/026921639500900306

Csikszentmihalyi, M. (1990). Flow: The Psychology of Optimal Experience. New York, NY: Harper and Row.

Csikszentmihalyi, M., and Larson, R. (1987). Validity and reliability of the experience-sampling method. J. Nerv. Ment. Dis. 175, 526-536. doi: 10.1097/00005053-198709000-00004

Edwards, J. R. (1994). The study of congruence in organizational behavior research: critique and a proposed alternative. Organ. Behav. Hum. Decis. Process. 58, 51-100. doi: 10.1006/obhd.1994.1029

Edwards, J. R., and Cooper, C. L. (1990). The person-environment fit approach to stress: recurring problems and some suggested solutions. J. Organ. Behav. 11, 293-307. doi: 10.1002/job.4030110405
Fanos, J. H., Gelinas, D. F., Foster, R. S., Postone, N., and Miller, R. G. (2008). Hope in palliative care: From narcissism to self-transcendence in amyotrophic lateral sclerosis. J. Palliative Care 11, 470-475. doi: 10.1089/jpm.2007.0098

Foley, G., Timonen, V., and Hardiman, O. (2014). Exerting control and adapting to loss in amyotrophic lateral sclerosis. Soc. Sci. Med. 101, 113-119. doi: 10.1016/j.socscimed.2013.11.003

Hammer, E. V., Häcker, S., Hautzinger, M., Meyer, T. D., and Kübler, A. (2008). Validity of the als-depression-inventory (adi-12): a new screening instrument for depressive disorders in patients with amyotrophic lateral sclerosis. J. Affect. Disord. 109, 213-219. doi: 10.1016/j.jad.2007.11.012

Iglesias, C., and Torgerson, D. (2000). Does length of questionnaire matter? a randomised trial of response rates to a mailed questionnaire. J. Health Serv. Res. Policy 5, 219-221. doi: 10.1177/135581960000500406

Jong-Meyer, R. D., Parthe, T., and Projektgruppe (2009). Einfluss von achtsamkeitsübung und dezentrierung auf rumination und spezifität autobiographischer erinnerungen. Z. Klin. Psychol. Psych. 38, 240-249. doi: 10.1026/16163443.38.4.240

Kenny, D. A., Bolger, N., and Korchmaros, J. D. (2003). Lower level mediation in multilevel models. Psychol. Methods 8, 115-128. doi: 10.1037/1082989X.8.2.115

King, S. J., Duke, M. M., and O'Connor, B. A. (2009). Living with amyotrophic lateral sclerosis/motor neurone disease (als/mnd): decision-making about 'ongoing change and adaptation'. J. Clin. Nurs. 18, 745-754. doi: 10.1111/j.13652702.2008.02671.x

Kocovski, N. L., Segal, Z. V., and Battista, S. R. (2009). "Mindfulness and psychopathology: problem formulation," in Clinical Handbook of Mindfulness, ed F. Didonna (New York, NY: Springer New York), 85-98.

Larson, R., and Csikszentmihalyi, M. (1983). "The experience sampling method," in Naturalistic Approaches to Studying Social Interaction, Vol. 15, ed H. T. Reis (San Francisco, CA: Jossey-Bass), 41-46.

Logroscino, G., Traynor, B. J., Hardiman, O., Chiò, A., Couratier, P., Mitchell, J. D., et al. (2008). Descriptive epidemiology of amyotrophic lateral sclerosis: new evidence and unsolved issues. J. Neurol. Neurosurg. Psychiatry 79, 6-11. doi: 10.1136/jnnp.2006.104828

Logroscino, G., Traynor, B. J., Hardiman, O., Chio, A., Mitchell, D., Swingler, R. J., et al. (2010). Incidence of amyotrophic lateral sclerosis in europe. J. Neurol. Neurosurg. Psychiatry 81, 385-390. doi: 10.1136/jnnp.2009.183525

Lulé, D., Häcker, S., Ludolph, A., Birbaumer, N., and Kübler, A. (2008). Depression and quality of life in patients with amyotrophic lateral sclerosis. Deutsch. Arztebl. Int. 105, 397-403. doi: 10.3238/arztebl.2008.0397

Lulé, D., Zickler, C., Häcker, S., Bruno, M. A., Demertzi, A., Pellas, F., et al. (2009). Life can be worth living in locked-in syndrome. Prog. Brain Res. 177, 339-351. doi: 10.1016/S0079-6123(09)17723-3

MacKinnon, D. P., Lockwood, C. M., Hoffman, J. M., West, S. G., and Sheets, V. (2002). A comparison of methods to test mediation and other intervening variable effects. Psychol. Methods 7, 83-104. doi: 10.1037/1082-989X.7.1.83

Martin, L. L., and Tesser, A. (1996). "Some ruminative thoughts," in Ruminative Thoughts, Vol. IX of Advances in Social Cognition, ed R. S. Wyer (Mahwah, NJ: Lawrence Erlbaum), 1-47.

Matuz, T., Birbaumer, N., Hautzinger, M., and Kübler, A. (2010). Coping with amyotrophic lateral sclerosis: an integrative view. J. Neurol. Neurosurg. Psychiatry 81, 893-898. doi: 10.1136/jnnp.2009.201285

McLeod, J. E., and Clarke, D. M. (2007). A review of psychosocial aspects of motor neurone disease. J. Neurol. Sci. 258, 4-10. doi: 10.1016/j.jns.2007.03.001

Montel, S., Albertini, L., and Spitz, E. (2012a). Coping strategies as related to medical and demographic data in amyotrophic lateral sclerosis. Acta Neurol. Scand. 125, 136-141. doi: 10.1111/j.1600-0404.2011.01513.x

Montel, S., Albertini, L., and Spitz, E. (2012b). Coping strategies in relation to quality of life in amyotrophic lateral sclerosis. Muscle Nerve 45, 131-134. doi: $10.1002 /$ mus. 22270

Nolen-Hoeksema, S. (1991). Responses to depression and their effects on the duration of depressive episodes. J. Abnorm. Psychol. 100, 569-582. doi: 10.1037/0021843X.100.4.569

Nolen-Hoeksema, S., Wisco, B. E., and Lyubomirsky, S. (2008). Rethinking rumination. Perspect. Psychol. Sci. 3, 400-424. doi: 10.1111/j.1745-6924.2008.00088.x

Nonnenmacher, S., Hammer, E. M., Lulé, D., Hautzinger, M., and Kübler, A. (2013). Psychische störungen und individuelle lebensqualität bei der chronisch progredient-terminalen erkrankung amyotrophe lateralsklerose (als). Z. Klin. Psychol. Psych. 42, 55-63. doi: 10.1026/1616-3443/a000186 
Pagnini, F. (2013). Psychological wellbeing and quality of life in amyotrophic lateral sclerosis: a review. Int. J. Psychol. 48, 194-205. doi: 10.1080/00207594.2012. 691977

Pagnini, F., Di Credico, C., Gatto, R., Fabiani, V., Rossi, G., Lunetta, C., et al. (2014a). Meditation training for people with amyotrophic lateral sclerosis and their caregivers. J. Altern. Complement. Med. 20, 272-275. doi: 10.1089/acm.2013.0268

Pagnini, F., Phillips, D., and Langer, E. (2014b). A mindful approach with end-oflife thoughts. Front. Psychol. 5:138. doi: 10.3389/fpsyg.2014.00138

Plahuta, J. M., McCulloch, B. J., Kasarskis, E. J., Ross, M. A., Walter, R. A., and McDonald, E. R. (2002). Amyotrophic lateral sclerosis and hopelessness: psychosocial factors. Soc. Sci. Med. 55, 2131-2140. doi: 10.1016/S02779536(01)00356-2

Rapkin, B. D., and Schwartz, C. E. (2004). Toward a theoretical model of qualityof-life appraisal: implications of findings from studies of response shift. Health Qual. Life Out. 2:14. doi: 10.1186/1477-7525-2-14

R Development Core Team (2011). R: A Language and Environment for Statistical Computing. Vienna: R Foundation for Statistical Computing.

Robbins, R. A., Simmons, Z., Bremer, B. A., Walsh, S. M., and Fisher, S. (2001). Quality of life in als is maintained as physical function declines. Neurology 56, 442-444. doi: 10.1212/WNL.56.4.442

Runyan, J. D., Steenbergh, T. A., Bainbridge, C., Daugherty, D. A., Oke, L., and Fry, B. N. (2013). A smartphone ecological momentary assessment - intervention app for collecting real-time data and promoting self-awareness. PLoS ONE 8:e71325. doi: 10.1371/journal.pone.0071325

Schmidt, J. A., Shernof, D. J., and Csikszentmihalyi, M. (2007). "Individual and situational factors related to the experience of flow in adolescence," in Oxford Handbook of Methods in Positive Psychology, Series in Positive Psychology, eds A. D. Ong and M. H. M. Van Dulmen (Oxford; New York, NY: Oxford University Press), 542-558.

Scollon, C. N., Prieto, C. K., and Diener, E. (2009). "Experience sampling: promises and pitfalls, strength and weaknesses," in Assessing Well-Being, Volume 39 of Social Indicators Research Series, ed E. Diener (Dordrecht: Springer), 157-180.

Selya, A. S., Rose, J. S., Dierker, L. C., Hedeker, D., and Mermelstein, R. J. (2012). A practical guide to calculating cohen's $\mathrm{f}$, a measure of local effect size, from proc mixed. Front. Psychol. 3:111. doi: 10.3389/fpsyg.2012.00111

Shiffman, S., Stone, A. A., and Hufford, M. R. (2008). Ecological momentary assessment. Annu. Rev. Clin. Psychol. 4, 1-32. doi: 10.1146/annurev. clinpsy.3.022806.091415
Smyth, J. M., and Stone, A. A. (2003). Ecological momentary assessment research in behavioral medicine. J. Happiness Stud. 4, 35-52. doi: 10.1023/A:102365 7221954

Snijders, T. A. B., and Bosker, R. J. (1993). Standard errors and sample sizes for two-level research. J. Educ. Behav. Stat. 18, 237-259. doi: 10.3102/10769986018 003237

Spearman, C. (1910). Correlation calculated from faulty data. Br. J. Psychol. 3, 271-295.

Stone, A. A., Shiffman, S. S., and DeVries, M. W. (2003). "Ecological momentary assessment," in Well-Being, eds D. Kahneman, E. Diener, and N. Schwarz (New York, NY: Russell Sage Foundation), 26-39.

Teuchmann, K., Totterdell, P., and Parker, S. K. (1999). Rushed, unhappy, and drained: an experience sampling study of relations between time pressure, perceived control, mood, and emotional exhaustion in a group of accountants. $J$. Occup. Health Psychol. 4, 37-54. doi: 10.1037/1076-8998.4.1.37

Tofighi, D., and MacKinnon, D. P. (2011). Rmediation: an r package for mediation analysis confidence intervals. Behav. Res. Methods 43, 692-700. doi: 10.3758/s13428-011-0076-x

Trail, M., Nelson, N. D., Van, J. N., Appel, S. H., and Lai, E. C. (2003). A study comparing patients with amyotrophic lateral sclerosis and their caregivers on measures of quality of life, depression, and their attitudes toward treatment options. J. Neurol. Sci. 209, 79-85. doi: 10.1016/S0022-510X(03)00003-0

Conflict of Interest Statement: The authors declare that the research was conducted in the absence of any commercial or financial relationships that could be construed as a potential conflict of interest.

Received: 07 May 2014; paper pending published: 29 May 2014; accepted: 19 June 2014; published online: 08 July 2014.

Citation: Real RGL, Dickhaus T, Ludolph A, Hautzinger M and Kübler A (2014) Wellbeing in amyotrophic lateral sclerosis: a pilot experience sampling study. Front. Psychol. 5:704. doi: 10.3389/fpsyg.2014.00704

This article was submitted to Psychology for Clinical Settings, a section of the journal Frontiers in Psychology.

Copyright (c) 2014 Real, Dickhaus, Ludolph, Hautzinger and Kübler. This is an openaccess article distributed under the terms of the Creative Commons Attribution License (CC BY). The use, distribution or reproduction in other forums is permitted, provided the original author(s) or licensor are credited and that the original publication in this journal is cited, in accordance with accepted academic practice. No use, distribution or reproduction is permitted which does not comply with these terms. 